\title{
La universidad y el poder en el Perú: Ios últimos cien años ${ }^{1}$
}

Artículos originales: SOCIOLOGÍA

Recibido: $13 / 09 / 2020$

Aprobado: 16/10/2020

Publicado: 05/02/2021
Nicolás Lynch Gamero

Universidad Nacional Mayor de San Marcos

nlynchg@unmsm.edu.pe

\begin{abstract}
RESUMEN
Este artículo trata del contrapunto entre la universidad y el poder en el Perú en los últimos cien años. Este asunto: la disputa de poder, en el mejor y el peor sentido de la palabra, es lo que va a determinar el decurso de esta relación. La universidad, en especial la universidad pública, tratando de ser un factor de democratización del país y el poder estatal, con actitudes cambiantes: la represión, el abandono, la privatización; buscando domesticar los impulsos rebeldes y cuestionadores de los universitarios. El portal de mira es la Universidad Nacional Mayor de San Marcos, desde su tradición, importancia y liderazgo, pero también desde su decadencia. Se trata, por lo demás, de un contrapunto irresuelto, que continúa una de las muchas interrogantes sobre el futuro de la democratización del Perú.
\end{abstract}

Palabras Clave: Perú; universidad; San Marcos; democratización; poder.

\section{University and power in Peru: the last hundred years}

\begin{abstract}
This article addresses the counterpoint between the university and political power in Peru during the last hundred years. This power dispute, in the best and worst sense, will determine the course of this relationship. The university, mainly the public university, tries to position itself as a democratizing factor in the country while state power embraces changing attitudes: repression, abandonment, and privatization, to tame the rebellious and questioning impulses of university professionals. We analyze this issue focusing on the Universidad Nacional Mayor de San Marcos, considering its tradition, importance, leadership, and decline. It is an unresolved counterpoint that continues among the many questions about the future of Peruvian democratization.
\end{abstract}

KeYwords: Perú; University; San Marcos; Democratization; Power.

1 Una versión abreviada de este artículo fue publicada en Pensamiento Universitario N. ${ }^{\circ} 19$, año 19. Octubre de 2020. 
E sta es una lectura de parte sobre un tema central, la universidad y el poder en el Perú. Para los que creen que la universidad peruana ha tenido en su historia nada más que un problema pedagógico o económico, o peor todavía de infiltración política de grupos radicales, les digo que no, el problema de la universidad ha sido y es un problema de relación con el poder. ¿Será capaz la sociedad peruana y su proyección estatal de darle a la universidad un lugar como espacio de producción democrática de conocimientos o por el contrario buscará someterla a un proyecto de reproducción social de alguna forma de dominación? Esto es lo que ha estado y está en juego en esta relación ańeja que ya cumplió un siglo desde el grito de la reforma y espero que no cumpla otro más en esta tensión.

La visión que se presenta sobre el tema en las siguientes líneas tiene dos sesgos: la universidad pública y la Universidad de San Marcos². La universidad pública si bien de crucial importancia en la mayor parte de la historia republicana ha devenido en los últimos veinte años en minoritaria en términos de la matrícula y la Universidad de San Marcos, la más grande y la de mayor importancia académica y política, ha perdido también importancia numérica y ha decaído en su peso específico en la vida universitaria y la vida nacional. Lo que no es óbice para que una visión desde la experiencia de sanmarquino y profesor de una universidad pública deje de interpelar la visión de la educación universitaria en el país.

Los últimos cien años de historia política del Perú está marcados por un contrapunto de la universidad con el poder, especialmente el poder del Estado. 1919, 1931, 1946, 1961, 1969, 1972, 1983, 1996, 2014; no son sino los momentos en este tiempo más largo, en el que los universitarios hemos ganado, perdido y también vuelto a ganar las libertades universitarias.

Desde la época de la lucha anti-oligárquica, esta ha sido la piedra de toque: nuestras libertades y la lucha por la democratización de la sociedad y el Estado. Cuando la universidad, en ese entonces casi toda pública, fue un faro de democratización, luchando por una apertura en acceso, cogobierno y contenidos educativos; hasta el período reciente de ofensiva

2 En todos los casos cuando se seńala la Universidad de San Marcos la referencia es a la Univesidad Nacional Mayor de San Marcos. por la privatización de la universidad, cuando la derecha neoliberal en el poder no solo quiere suprimir rebeldías sino también hacer de la educación, especialmente la educación universitaria, un mecanismo de reproducción de su dominación social. Para esto último, tiene que dejar de lado todo lo que sea democracia y cambiar la lógica de la institución pugnando que pase de proveer un derecho a administrar una mercancía.

\section{La universidad y la lucha anti-oligárquica}

El período de la lucha anti-oligárquica en el movimiento universitario peruano va de 1919 a 1961. 1919 es un año no sólo de partida sino emblemático para los universitarios peruanos, fue el año de recepción de las noticias del movimiento de reforma universitaria ocurrido en Córdoba, Argentina el año anterior, noticias que trajo el socialista Alfredo Palacios que vino de visita al Perú, en gira reseñada en el momento y recordada después por José Carlos Mariátegui (1970), en sus múltiples referencias a la reforma. Las novedades se convirtieron en movimiento y los universitarios lograron del naciente gobierno de Augusto B. Leguía las primeras reivindicaciones de la reforma. Por ello, 1919 fue también un punto de ruptura con la antigua universidad oligárquica en una agonía interminable que no se termina hasta hoy.

Mientras tanto, la iglesia católica con el dinero y en los terrenos de José de la Riva Agüero y Osma, crea en 1917 la Pontificia Universidad Católica del Perú (PUCP), de poca importancia en el período de lucha anti oligárquica, como contrapeso ideológico a las universidades públicas y especialmente a San Marcos, pero que adquirió peso en la etapa siguiente de desarrollo universitario.

Sin embargo, las primaveras en la lucha anti oligárquica fueron cortas. Esta primera, concedida por Leguía, duró hasta que este cambió la constitución en 1923 para poder reelegirse y los universitarios se movilizaron inútilmente para impedirlo, dejando muertos en las calles. El movimiento, sin embargo, no dejó de tener frutos. Lo dirigió Víctor Raúl Haya de la Torre, consagrándose como el nuevo líder insurgente, en una jornada que, nuevamente Mariátegui (1970), denominaría «el bautismo histórico de la nueva generación». Las primaveras posteriores fueron más cortas 
y reprimidas por dictaduras militares, como en los casos de 1931 y 1948 en las que iniciales reformas fueron cortadas por el fin de las aperturas políticas que les dieron vida, con lo que el programa de la reforma pasó a ser un ideario siempre en ciernes. Ello llevó a Augusto Salazar Bondy, el gran filósofo de la educación peruana en la segunda mitad del siglo XX, a decir en 1957 que el movimiento universitario se había preocupado más por las banderas políticas de autonomía, acceso y cogobierno que por el desarrollo de la calidad académica, cuyo deterioro ya se notaba en las universidades públicas.

\section{El abandono del Estado y la masificación sin proyecto}

En 1961 hubo un giro a tomar en cuenta, se aprobó una nueva ley universitaria, la 13417, en las condiciones del gobierno oligárquico de Manuel Prado elegido con el respaldo del Partido Aprista, todavía proscrito. La ley incluyó, a pesar de la dura resistencia derechista, el cogobierno universitario. Esto último motivó la ruptura de la Facultad de Medicina de San Marcos, fundando los profesores que se oponían a la participación estudiantil la Universidad Peruana Cayetano Heredia, principalmente dedicada a las ciencias médicas. Sin embargo, la ley universitaria 13417 fue importante porque a partir de ella se estableció el cogobierno en las universidades públicas y las privadas de comunidad universitaria (llamadas hoy eufemísticamente «asociativas»). Pero este cogobierno acentuó la politización que tomó como bandera la masificación de la educación universitaria, una «masificación sin proyecto» (Lynch 1990, 2006) porque era un reclamo sin un proyecto de universidad de por medio. Este reclamo de masificación se dio en medio de una aguda pugna, especialmente violenta, en el movimiento universitario entre el Partido Aprista, que ya había girado a una "convivencia»" con la oligarquía y las agrupaciones de izquierda, principalmente maoístas. La izquierda radical sale triunfante de este enfrentamiento, pero con un alto costo en

3 Se denominó convivencia en la política peruano al período de gobierno de Manuel Prado (1956-1962), el último presidente de la vieja oligarquía, que accedió al poder gracias a un «pacto de no agresión» con el Partido Aprista que continuó proscrito hasta 1962, pero dejó gobernar a Prado. términos de destrucción de los campus y de la calidad de la enseñanza, lo que tiene como consecuencia la migración de los mejores catedráticos a universidades privadas y/o extranjeras.

Esta lucha, tanto es sus querellas intestinas como en el enfrentamiento a sucesivos gobiernos, tuvo como consecuencia el abandono de la universidad ${ }^{4}$, me refiero específicamente a la pública, por parte del Estado. Un abandono, ciertamente con varios paréntesis, pero abandono a fin y al cabo, de lo que consideró primero una casa rebelde y después una casa incendiada ${ }^{5}$, inútil siquiera para promover la reproducción de su dominación social. Esta etapa de abandono duró entre 1961 y 1996, en que la dictadura de Alberto Fujimori da el Decreto Legislativo 882, que permite la creación de las universidades con fines de lucro. El abandono tiene como característica central el ahogamiento financiero de las universidades públicas, mientras se permite su multiplicación, así como la primera ampliación en el número de las universidades privadas. Sobre esto último es interesante resaltar que las dos universidades de importancia en la época, la Pontificia Universidad Católica y la Universidad Cayetano Heredia, reciben en el período entre el 50 y el $70 \%$ de su presupuesto del Estado. Precisando la característica de este abandono diría que se trata de incuria, en el sentido de desprecio de los poderes de turno por la universidad, específicamente la pública. Zenón de Paz (2003) señala que este pasaje de la universidad como espacio de democratización anti oligárquico al abandono de la institución por el Estado y la radicalización extrema del movimiento estudiantil serán el pasaje del espacio de todos a la tierra de nadie.

El primer paréntesis son las dos leyes que da el gobierno militar reformista del general Juan Velasco, la primera en 1969 y la segunda en 1972. Ambas, aun-

4 Soy un testigo de ese abandono porque ingresé como estudiante a la Universidad de San Marcos en 1971 para estudiar sociología y egresé, estudiando en condiciones en extremo precarias, en 1976. Di cuenta de esta situación en mi libro «Los jóvenes rojos de San Marcos» (1990).

5 La metáfora de incendio no es gratuita, proviene de una incursión que hice al Congreso de la República a mediados de la década de 1990, junto con una delegación de profesores de San Marcos, para reclamar más presupuesto para la universidad. Recuerdo un intercambio con un asesor del Ministro de Economía de la época que refiriéndose al monto que nos estaban asignando me espetó: «no le parece sufuciente dinero para un seguro contra incendios», refiriéndose a que lo que se le daba a la universidad no era un presupuesto para el financiamiento de una institución académica sino una cantidad de dinero para mantener a los universitarios tranquilos. 
que especialmente la primera, buscan restringir el cogobierno universitario y promover una reorganización de la estructura académica con el fin de modernizar la universidad y articularla con un proyecto de desarrollo nacional que buscaba encarnar ese gobierno militar (Germaná 2003). La iniciativa es ferozmente resistida por el movimiento estudiantil en todo el país y queda vigente como ley pero en la práctica paralizada. El segundo, es el de la ley 23733 de 1983, durante el gobierno elegido del conservador Fernando Belaúnde. A esta ley Juan Abugattás se refirió como un "Córdoba tardío ${ }^{6}$, porque intentaba restaurar las instituciones del movimiento reformista inicial que parcialmente habían sido logradas en el medio siglo anterior, especialmente el cogobierno universitario, el régimen de facultades y la Asamblea Nacional de Rectores. Sin embargo, como afirmaba el mismo Abugattás, en un momento de desarrollo del conocimiento en el que las demandas eran mayores que a principios del siglo XX para el desarrollo académico del mismo. El impacto restaurador, incluido el de la antigua Asamblea Nacional de Rectores, fue el inmovilismo.

Un elemento que agudizó el abandono y el inmovilismo, debilitando aún más a la universidad pública y facilitando la privatización posterior, fue la guerra interna que se libró entre 1980 y 1992 entre las fuerzas armadas y policiales y los grupos alzados en armas: Sendero Luminoso y el Movimiento Revolucionario Túpac Amaru. Esta guerra tuvo como uno de sus escenarios los campus de varias universidades públicas ${ }^{7}$, pero fue utilizado por los grandes medios y los políticos de derecha para satanizar al conjunto tildándolos de nidos de terroristas. La consecuencia, especialmente luego del golpe de Estado del 5 de abril de 1992, momento en que Fujimori se convierte en dictador, fue que varios de estos campus fueron ocupados militarmente e incluso algunos donde la incidencia había sido menor pero cuyos movimientos tenían fama de rebeldes, como la Universidad de San Marcos, también intervenidos por el gobierno central que suspendió su autonomía académica y administrativa hasta la restauración de la democracia el año $2000^{8}$. En esta

6 Comunicación personal de Juan Abugattás con el autor.

7 Este fue el caso de la Universidad Nacional de Ingeniería y la Universidad Nacional de Educación "La Cantuta», en Lima; de la Universidad Nacional San Cristóbal de Huamanga en Ayacucho y de la Universidad Nacional del Centro en Huancayo.

8 La Universidad de San Marcos fue ocupada por el Ejército Peruano entre 1992 y el 2000 e intervenida por el Poder Ejecutivo, retiran- ocupación militar y posterior intervención no solo jugó un papel la persecución a los grupos subversivos, sino la bronca acumulada contra la universidad pública y las ganas de darle un escarmiento por su actitud contestataria.

\section{La ola privatizadora}

A partir de 1996 tenemos la actual ola privatizadora cuya puerta se abre con el DL 882. Entre 1996 y el 2012 se da la primera fase de la privatización que se caracteriza por la fundación masiva de universidades privadas con fines de lucro y tiene como resultado la existencia al 2012, ańo en que se declara la moratoria de creación de nuevas universidades, 142 universidades, 51 públicas y 91 privadas, de las últimas 51 son con fines de lucro (ANR 2012, SUNEDU 2017). Lo curioso de este vendaval privatizador es que ocurre sin cambios de fondo sino tan solo parches de la ley 23733. Esta fundación masiva de universidades con dueño, cuestión que antes de 1996 estaba prohibida en el Perú, constituye la etapa de mayor envilecimiento de la educación universitaria en el Perú. Como dijo un amigo catedrático, cualquiera enseñó cualquier cosa en cualquier parte. El argumento de que estas universidades privadas llenaron una demanda que no era satisfecha por las universidades públicas es falaz, ya que esto sucedió por el abandono de estas por el Estado. Se trató entonces de usar el abandono que venía de atrás para que la dictadura de Fujimori, como abanderada de un proyecto neoliberal, abriera el mercado de la educación y en especial el mercado universitario a la inversión privada.

La creación de decenas de universidades de pésima calidad que arrinconaban a las universidades públicas y también a las privadas más antiguas, despertó nuevamente al movimiento reformista. Desde varias universidades: la Universidad de San Marcos, la Universidad Nacional de Ingeniería (UNI), la PUCP, la Universidad Cayetano Heredia; surgió un grupo de profesores, algunos ex rectores de sus universidades,

do formalmente su autonomía entre 1995 y 2000. En los primeros meses de la intervención militar los soldados vigilaban las puertas del campus y se paseaban por los pasillos de las facultades, o sea, que durante un tiempo dimos clases vigilados por soldados con fusiles de guerra. Luego de ese primer momento se replegaron a su cuartel establecido en el gimnasio de la Ciudad Universitaria. 
que decidieron juntarse para levantar una alternativa reformista al caos privatizador. Teniendo como antecedentes dos proyectos de ley universitaria presentados por el Ministerio de Educación en los años 2002 y 2005, el grupo recorrió el Perú haciendo cerca de 40 conferencias y talleres en universidades públicas y privadas, entre los ańos 2004 y 2007. Ello culminó en un proyecto de ley universitaria que fue aprobado por la Comisión de Educación del Congreso de la República el año 2009; este proyecto de ley es el antecedente más inmediato de la ley 30220 que se aprobaría el 2014.

Sin embargo, de manera relativamente silenciosa, avanzaba otro grupo - me refiero a los tecnócratas neoliberales afincados en el Ministerio de Economía y Finanzas (MEF) - hacia la toma de los ministerios dedicados a los sectores sociales. En una situación de democracia sin partidos (Tanaka 2005) y más todavía de democracia sin democratización (Lynch 2009), en la que los elencos políticos han rotado a velocidad inusitada manchados por sucesivos escándalos de corrupción, ha llegado a ser muy importante el papel de la tecnocracia neoliberal en la pirámide de poder en el Perú. Los tecnócratas neoliberales, como señala Francisco Durand (2019) han pasado a ser el elemento permanente en el estado peruano, los garantes de la estabilidad en esta era neoliberal, expresando los intereses de los grandes propietarios y convirtiéndose en los administradores del aparato estatal más allá de los cambios de gobierno. Esta situación se remonta a la dictadura de Alberto Fujimori a la que la naciente tecnocracia sirvió lealmente y transita de dictadura a democracia, acomodándose sin inmutarse con los siguientes presidentes. Hay dos gobiernos que favorecen especialmente a esta tecnocracia que son los de Alan García (2006-2011) y Ollanta Humala (2011-2016). En estos dos, pero en especial en el gobierno de Humala se produce el tránsito al control del Ministerio de Educación. El afianzamiento definitivo se produce con el ministerio que encabeza Jaime Saavedra, entre el 2013 y el 2016 y en el confluyen tecnócratas que vienen del MEF, así como de ONG ligadas a los organismos financieros internacionales como el Grupo de Análisis para el Desarrollo (GRADE) de donde proviene el propio Saavedra y también el actual ministro Martín Benavides; se suman técnicos del Instituto de Estudios Peruanos, y de universidades privadas como la propia PUCP y la
Universidad del Pacífico. No es casual que esto signifique el desplazamiento de funcionarios con una visión distinta que provenían de la Universidad Nacional de Educación «La Cantuta» de la Universidad de San Marcos y de otras universidades públicas.

Pero la oportunidad para tomar el control de la educación universitaria se da el año 2014. En el momento en que, ya con un proyecto listo, el congresista Daniel Mora, que lidera la confección de una nueva ley universitaria y había recogido los aportes de diversos sectores reformistas, pero especialmente los del proyecto del 2009, estaba juntando los votos para conseguir en el pleno del Congreso la aprobación de la ley. En ese momento se acerca el entonces ministro Jaime Saavedra y le ofrece un trato: los votos de la bancada oficialista, que controlaba el gobierno de Ollanta Humala, a cambio de algunas modificaciones en el proyecto terminado. Mora, ante la eventualidad de no aprobar nada, acepta y proceden las modificaciones. Estas fueron centralmente dos: el reemplazo del capítulo 2 del proyecto, donde se legislaba lo relativo al órgano regulador, reemplazando el Consejo Nacional de Universidades, concebido como una regulación surgida democráticamente de las propias universidades, por la Superintendencia Nacional de Educación Superior Universitaria (SUNEDU) un órgano regulador extrauniversitario. Asimismo, borra toda mención a derogar el DL 882, por más que, en lo que concierne a universidades, es reemplazado por la nueva ley.

Saavedra y su grupo obtienen lo que querían tomando por asalto una ley en cuya elaboración no habían participado hasta el momento final y usan esta oportunidad para desarrollar un proyecto mayor: la segunda fase de la privatización universitaria, hoy en curso en el Perú. Sería un error quedarnos en la anécdota y creer que todo se trata de coincidencias. Estos tecnócratas se apropian del trabajo reformista y le agregan su sazón porque tienen el poder político para hacerlo, en su momento el respaldo del gobierno de Humala, ya totalmente entregado a la derecha neoliberal, pero más allá el respaldo de la tecnocracia que administra el Estado peruano desde la década de 1990, sus ramificaciones con el poder económico y con los organismos internacionales, especialmente el Banco Mundial y el Banco Interamericano de Desarrollo. Además, han tenido la audacia de poner las cosas al revés y tener éxito, por el apoyo mediático que ostentan, llamando «reforma» a lo que en el fondo 
es una "contrarreforma» de la educación universitaria. Para ello, fue crucial que fueran conscientes del fracaso de la primera ola privatizadora y la necesidad de ir a una segunda desarrollada con criterios distintos.

Este grupo de tecnócratas introdujeron la SUNEDU y han interpretado la ley 30220 de manera tal, que les permita el control político de las universidades, para colocarlas en una lógica privatizadora y ponerlas al servicio del modelo neoliberal, es decir incluirlas a todas, de una u otra manera, en un mercado de educación universitaria. Para esto han interpretado abusivamente la rectoría que el Estado según la ley ejerce sobre el sistema universitario, pasando de la elaboración de políticas generales a la implementación operativa de las mismas, tanto a través de una Dirección de Educación Superior del Ministerio de Educación como de la propio SUNEDU. Esta interpretación abusiva de la ley los lleva a pretender aprobar las autorizaciones de funcionamiento (o licencias como dicen ellos) de las universidades no sólo por sus características generales sino llegando al análisis del detalle académico y administrativo, para convertirse de esta forma en la autoridad suprema del mundo universitario. De esta manera, se transforman en violadores reiterados de la autonomía universitaria, objetivo central de su propósito, porque así debilitan el cogobierno y la gratuidad de la enseñanza en las públicas y establecen una espada de Damocles para todas las demás, señalando que quien no cumpla con las directivas del regulador puede quedar fuera de juego.

La necesidad de la regulación del conjunto del sistema universitario era una idea que había surgido a principios del 2000 en los primeros proyectos de reforma que se hicieron, debido a la inoperancia de la Asamblea Nacional de Rectores, un mecanismo de coordinación que no había funcionado ni siquiera como tal. Además, un brazo de esta la Comisión de Autorización de Funcionamiento de Universidades (CONAFU) había sido el organismo que autorizó la avalancha de universidades con fines de lucro a partir del DL 882. Sin embargo, en todos los planteamientos, de una u otra forma, se concebía un organismo que nacía de las universidades. En ningún caso un organismo ajeno a ellas. Por la sencilla razón que sólo teniendo ligazón con la universidad como institución el organismo regulador tendría legitimidad para ejercer su función. Los tecnócratas a cargo de diseñar la SUNEDU han tratado de maquillar este incon- veniente poniendo a profesores universitarios en el mismo. Sin embargo, estos profesores son elegidos en un concurso que convoca el Ministerio de Educación y es calificado por el Consejo Nacional de Educación, elegido por el mismo ministerio. Por último, el presidente de la SUNEDU, es designado por el propio ministro del sector. Todo entonces queda en casa.

La privatización en el caso de las universidades públicas significa continuar con la política de subfinanciamiento que venía implementado el Estado, dándoles año a año significativamente menos dinero del que piden y mucho menos del que necesitan, así sus niveles de ejecución presupuestal sean óptimos. Esto ha significado reducir el financiamiento directo que da el Tesoro Público y promover el aumento de los recursos directamente recaudados, de manera tal que estas universidades se conviertan en modestas proveedoras de un recurso cada vez más empobrecido. Como burla, porque no queda otra palabra para calificarlo, ponen a disposición de estas universidades pequeñas cantidades de dinero todos los años que se llevan las que se porten mejor. A esto se suma la creación de programas perversos como Beca 18, un programa del Estado con un presupuesto aproximadamente del $30 \%$ del presupuesto total que da el Estado a las 51 universidades públicas, destinado a que estudiantes pobres estudien en la universidad (Portal de Transparencia. Gobierno del Perú 20142020). En sus primeros años este programa fue en un $90 \%$ para universidades privadas, aunque debido a lo escandaloso de la situación poco a poco ha ido derivando más a universidades públicas. Este dinero es en realidad el que el Estado debe destinar a mejorar el presupuesto de las públicas, aunque siguiendo las recomendaciones del Banco Mundial prefiere destinarlo a financiar la demanda antes que la oferta. Se establece así una lógica privatizadora, como bien señala para diversos casos en América Latina Fernanda Safocarda (2019), de los recursos del Tesoro Público que apunta a reducir cada vez más el financiamiento del Estado a las universidades nacionales.

Asimismo, la SUNEDU como ente regulador actúa de 2016 en adelante y en estos años ha tenido buena prensa por la labor cumplida en el cierre de una cantidad importante de instituciones de educación superior universitaria, 44 universidades y dos escuelas de posgrado, de las universidades 43 privadas con fines de lucro y una pública (SUNEDU 2020). 
Ha capitalizado, en este sentido el descontento frente al pésimo servicio que ofrecían buena parte de las universidades con fines de lucro. Sin embargo, al mismo tiempo ha desplegado su actividad revisando hasta los planes de estudio de las distintas facultades, en clara violación de la autonomía y la libertad de cátedra. Para esta labor cuenta, desafortunadamente, con autoridades genuflexas lastradas por la mediocridad en muchas universidades, que carecen de la fuerza personal e institucional para enfrentarlos. Se trata, sin embargo, de una instancia seriamente cuestionada en el Perú, aunque este cuestionamiento venga principalmente de los dueños de las universidades con fines de lucro cuya autorización ha sido denegada, es decir de los mercaderes de la educación surgidos al amparo de la primera ola privatizadora. El otro cuestionamiento, de los universitarios que levantan el programa de la reforma, está todavía subordinado al primero, aunque va ganando simpatía en diferentes campus. Empero, el ambiente político general no es favorable para estos tecnócratas neoliberales, porque el modelo que los ha procreado está en crisis y la estructura de poder de la que forman parte también. Esto es un buen augurio para una futura renovación de la universidad peruana, ojalá que esta vez sí en un sentido perdurable.

\section{Conclusión}

Tenemos entonces que, desde la lucha anti oligárquica, pasando por el abandono del Estado y la radicalización estudiantil, hasta las olas privatizadoras del neoliberalismo, no logra estabilizarse una relación entre el poder estatal y la universidad en el Perú. Quizás la ilusión del último quinquenio con la SUNEDU haya sido uno de los esfuerzos más importantes del Estado, pero la insistencia en hacerlo desde afuera, tal cual actúa el Estado peruano como un poder ajeno a la sociedad lo condene al fracaso. A la luz de esta historia, es difícil imaginarse la convivencia entre una universidad reformada con una ausencia de democratización en la sociedad. Es posible que el único camino sea un esfuerzo paralelo de democratización en el que la universidad encuentre un entorno favorable para volver a encontrarse con el país del que forma parte.

\section{Bibliografía}

Asamblea Nacional de Rectores (2012). Datos Estadísticos Universitarios. Lima.

De PAz, Zenón (2003). Universidad y democracia. Nosotros No.2 Lima-Perú, agosto.

Durand, Francisco (2019). La captura del Estado en América Latina. Reflexiones teóricas. Lima: OXFAM, Fondo Editorial Pontificia Universidad Católica del Perú.

Germaná, César (2003). La universidad peruana en proceso de transición. Medicina No. 8. Trujillo, marzo.

Lynch, Nicolás (1990). Los jóvenes rojos de San Marcos. Lima: El zorro de abajo ediciones.

LYNCH, Nicolás (2006). La segunda reforma universitaria. Los últimos de la clase. Aliados, adversarios y enemigos de la reforma educativa en el Perú. Lima: Fondo Editorial de la Universidad Nacional Mayor de San Marcos.

LyNCH, Nicolás (2009). El argumento democrático sobre América Latina. La excepcionalidad peruana en perspectiva comparada. Lima: Fondo editorial de la Univesidad Nacional Mayor de San Marcos.

Mariátegui, José Carlos (1970). El proceso de la instrucción pública. 7 ensayos de interpretación de la realidad peruana. 18 edición. Lima: Empresa Editora Amauta.

Portal de Transparecia. Gob.pe https://www.transparencia.gob.pe/enlaces/pte_transparencia_enlaces.aspx?id_ entidad=14184\&id_tema=5\&ver=\#.XvKXcpMzZTY

SAFOCARDA, Fernanda (2019). Introducción. Tendencias de privatización y mercantilización de la Universidad en América Latina. Los casos de Argentina, Chile, Perú y la República Dominicana. Buenos Aires: Internacional de la Educación, Instituto de Estudios y Capacitación- CONADU.

Salazar Bondy, Augusto (1957). Mitos, dogmas y postulados de la Reforma Universitaria. Lima: Facultad de Letras. Universidad Nacional Mayor de San Marcos.

Superintendencia Nacional de Educación Superior UNIVERSITARIA (2017). Informe bienal sobre la realidad universitaria. Lima.

Superintendencia Nacional de Educación Superior UnIVERsitaria (2020). Estado de licenciamiento de las universisdades. https://www.sunedu.gob.pe/ avances-licenciamiento/

Tanaka, Martín (2005). Democracia sin partidos: Perú, 2000-2005. Los problemas de representación y las propuestas de reforma política. Lima: Instituto de Estudios Peruanos. 